\title{
A Fixed Point Theorem for Contraction Type Maps in Partially Ordered Metric Spaces and Application to Ordinary Differential Equations
}

\author{
M. Eshaghi Gordji, ${ }^{1}$ H. Baghani, ${ }^{1}$ and G. H. Kim ${ }^{2}$ \\ ${ }^{1}$ Department of Mathematics, Semnan University, P.O. Box 35195-363, Semnan, Iran \\ ${ }^{2}$ Department of Mathematics, Kangnam University, Gyeonggi, Yongin 446-702, Republic of Korea \\ Correspondence should be addressed to G. H. Kim, ghkim@kangnam.ac.kr
}

Received 21 November 2011; Accepted 20 December 2011

Academic Editor: Mingshu Peng

Copyright (C) 2012 M. Eshaghi Gordji et al. This is an open access article distributed under the Creative Commons Attribution License, which permits unrestricted use, distribution, and reproduction in any medium, provided the original work is properly cited.

We present a fixed point theorem for generalized contraction in partially ordered complete metric spaces. As an application, we give an existence and uniqueness for the solution of a periodic boundary value problem.

\section{Introduction}

The contraction mapping theorem and the abstract monotone iterative technique are well known and are applicable to a variety of situations. Recently, there has been a trend to weaken the requirement on the contraction by considering metric spaces endowed with partial order (see [1-7]). It is of interest to determine if it is still possible to establish the existence of a unique fixed point assuming that the operator considered is monotone in such a setting. Such a fixed point theorem is useful, for example, in establishing the existence of a unique solution to periodic boundary value problems, besides many others.

That approach was initiated by Ran and Reurings in [8], where some applications to matrix equations were studied. This fixed point theorem was refined and extended in $[7,9]$ and applied to the periodic boundary value problem in the monotone case. In this paper, we consider a special case of the following periodic boundary value problem

$$
\begin{gathered}
u^{\prime}(t)=f(t, u(t)) \quad \text { if } t \in I=[0, T] \\
u(0)=u(T)
\end{gathered}
$$

where $T>0$ and $f: I \times \mathbb{R} \rightarrow \mathbb{R}$ is continuous function. 
Definition 1.1. A lower solution for (1.1) is a function $u \in C^{1}(I, \mathbb{R})$ such that

$$
\begin{gathered}
u^{\prime}(t) \leq f(t, u(t)) \quad \text { for } t \in I=[0, T] \\
u(0) \leq u(T) .
\end{gathered}
$$

Very recently, Harjani and Sadarangani [4] proved the following existence theorem.

Theorem 1.2. Consider problem (1.1) with $f: I \times \mathbb{R} \rightarrow \mathbb{R}$ is continuous and suppose that there exists $\lambda>0$ such that for $x, y \in \mathbb{R}$ with $y \geq x$

$$
0 \leq f(t, y)+\lambda y-[f(t, x)+\lambda x] \leq \lambda \phi(y-x)
$$

where $\phi:[0, \infty) \rightarrow[0, \infty)$ can be written by $\phi(x)=x-\psi(x)$ with $\psi:[0, \infty) \rightarrow[0, \infty)$ continuous increasing positive in $(0, \infty), \psi(0)=0$ and $\lim _{t \rightarrow \infty} \psi(t)=\infty$. Then the existence of a lower solution of (1.1) provides the existence of a unique solution of (1.1).

In Section 2, we prove a new fixed point theorem in partially ordered complete metric spaces. In Section 3, existence of a unique solution for problem (1.1) is obtained under suitable conditions.

\section{Fixed Point Theorem}

Let $S$ denote the class of those functions $\alpha:[0, \infty) \rightarrow[0,1)$ which satisfies the condition

$$
\limsup _{s \rightarrow t^{+}} \alpha(s)<1, \quad \forall t \in[0, \infty)
$$

We prove the main theorem of the paper as follows.

Theorem 2.1. Let $(X, \preccurlyeq)$ be a partially order metric space that there exists a metric $d$ in $X$ such that $(X, d)$ is a complete metric space. Let $f: X \rightarrow X$ be an increasing mapping such that there exists $x_{0} \in X$ with $x_{0} \preccurlyeq f\left(x_{0}\right)$. Suppose that there exists $\alpha \in S$ such that

$$
d(f(x), f(y)) \leq \alpha(d(x, y)) d(x, y)
$$

for all comparable $x, y \in X$. If

$$
f \text { is continuous }
$$

or

$$
\text { if an increasing sequence }\left\{x_{n}\right\} \rightarrow x \text { in } X \text {, then } x_{n} \preccurlyeq x, \forall n \in \mathbb{N} \text {. }
$$


Besides, if

for each $x, y \in X$, there exists $z \in X$ which is comparable to $x$ and $y$,

then $f$ have a unique fixed point.

Proof. We first show that $f$ has a fixed point. Since $x_{0} \preccurlyeq f\left(x_{0}\right)$ and $f$ is increasing function, we obtain by induction that

$$
x_{0} \preccurlyeq f\left(x_{0}\right) \preccurlyeq f^{2}\left(x_{0}\right) \preccurlyeq f^{3}\left(x_{0}\right) \preccurlyeq \cdots \preccurlyeq f^{n}\left(x_{0}\right) \preccurlyeq f^{n+1}\left(x_{0}\right) \cdots
$$

Put $x_{n}=f^{n}\left(x_{0}\right), n=1,2, \ldots$. Since $x_{n} \preccurlyeq x_{n+1}$ for each $n \in \mathbb{N}$ then by (2.2)

$$
\begin{aligned}
d\left(x_{n+1}, x_{n+2}\right) & =d\left(f^{n+1}\left(x_{0}\right), f^{n+2}\left(x_{0}\right)\right) \\
& \leq \alpha\left(d\left(x_{n}, x_{n+1}\right)\right) d\left(x_{n}, x_{n+1}\right) \\
& \leq d\left(x_{n}, x_{n+1}\right) .
\end{aligned}
$$

And so the sequence $\left\{d\left(x_{n+1}, x_{n}\right)\right\}$ is nonincreasing and bounded below. Thus there exists $\tau \geq 0$ such that $\lim _{n \rightarrow \infty} d\left(x_{n+1}, x_{n}\right)=\tau$. Since $\limsup _{s \rightarrow \tau^{+}} \alpha(s)<1$ and $\alpha(\tau)<1$ then there exist $r \in[0,1)$ and $\epsilon>0$ such that $\alpha(s)<r$ for all $s \in[\tau, \tau+\epsilon]$. We can take $v \in \mathbb{N}$ such that $\tau \leq d\left(x_{n+1}, x_{n}\right) \leq \tau+\epsilon$ for all $n \in \mathbb{N}$ with $n \geq v$. Then since

$$
d\left(x_{n+1}, x_{n+2}\right) \leq \alpha\left(d\left(x_{n}, x_{n+1}\right)\right) d\left(x_{n}, x_{n+1}\right) \leq r d\left(x_{n}, x_{n+1}\right),
$$

for all $n \in \mathbb{N}$ with $n \geq v$ we have

$$
\sum_{n=1}^{\infty} d\left(x_{n}, x_{n+1}\right) \leq \sum_{n=1}^{v} d\left(x_{n}, x_{n+1}\right)+\sum_{n=1}^{\infty} r^{n} d\left(x_{v}, x_{v+1}\right)<\infty,
$$

and hence $\left\{x_{n}\right\}$ is a Cauchy sequence. Since $X$ is complete, $\left\{x_{n}\right\}$ converges to some point $z \in X$. To prove that $z$ is a fixed point of $f$, if $f$ is a continuous, then

$$
z=\lim _{n \rightarrow \infty} x_{n}=\lim _{n \rightarrow \infty} f^{n}\left(x_{0}\right)=\lim _{n \rightarrow \infty} f^{n+1}\left(x_{0}\right)=f\left(\lim _{n \rightarrow \infty} f^{n}\left(x_{0}\right)\right)=f(z) ;
$$

hence $z=f(z)$. If case (2.4) holds then

$$
\begin{aligned}
d(f(z), z) & \leq d\left(f(z), f\left(x_{n}\right)\right)+d\left(f\left(x_{n}\right), z\right) \\
& \leq \alpha\left(d\left(x_{n}, z\right)\right) d\left(x_{n}, z\right)+d\left(x_{n+1}, z\right) \\
& \leq d\left(x_{n}, z\right)+d\left(x_{n+1}, z\right) .
\end{aligned}
$$


Since $d\left(x_{n}, z\right) \rightarrow 0$ then we get $f(z)=z$. To prove the uniqueness of the fixed point, let $y$ be another fixed point of $f$. From (2.5) there exists $x \in X$ which is comparable to $y$ and $z$. Monotonicity implies that $f^{n}(x)$ is comparable to $f^{n}(y)=y$ and $f^{n}(z)=z$ for $n=0,1, \ldots$. Moreover,

$$
\begin{aligned}
d\left(z, f^{n}(x)\right) & =d\left(f^{n}(z), f^{n}(x)\right) \\
& \leq \alpha\left(d\left(f^{n-1}(z), f^{n-1}(x)\right)\right) d\left(f^{n-1}(z), f^{n-1}(x)\right) \\
& \leq d\left(f^{n-1}(z), f^{n-1}(x)\right) \\
& =d\left(z, f^{n-1}(x)\right)
\end{aligned}
$$

Consequently, the sequence $\zeta_{n}^{z}:=d\left(z, f^{n}(x)\right)$ is nonnegative and decreasing and so $\lim _{n \rightarrow \infty} d\left(z, f^{n}(x)\right)=\zeta_{z} \in \mathbb{R}$. Similarly we can show that the sequence $\zeta_{n}^{y}:=d\left(y, f^{n}(x)\right)$ is nonnegative and decreasing and so $\lim _{n \rightarrow \infty} d\left(y, f^{n}(x)\right)=\zeta_{y} \in \mathbb{R}$. Now similarly the above method we can choose $r_{1}, r_{2}$ in $[0,1)$ and $\tau_{1} \in \mathbb{N}$ such that

$$
\begin{aligned}
& d\left(z, f^{n}(x)\right) \leq \alpha\left(d\left(z, f^{n-1}(x)\right)\right) d\left(z, f^{n-1}(x)\right) \leq r_{1} d\left(z, f^{n-1}(x)\right) \\
& d\left(y, f^{n}(x)\right) \leq \alpha\left(d\left(y, f^{n-1}(x)\right)\right) d\left(y, f^{n-1}(x)\right) \leq r_{2} d\left(y, f^{n-1}(x)\right)
\end{aligned}
$$

for all $n \in \mathbb{N}$ with $n>\tau_{1}$. Finally

$$
d(z, y) \leq d\left(z, f^{n}(x)\right)+d\left(f^{n}(x), y\right) \leq r_{1}^{n-\tau_{1}} d\left(z, f^{\tau_{1}} x_{0}\right)+r_{2}^{n-\tau_{1}} d\left(y, f^{\tau_{1}} x_{0}\right)
$$

for all $n \in \mathbb{N}$ with $n>\tau_{1}$. Therefor if in (2.14) taking $n \rightarrow \infty$ yields $d(z, y)=0$.

\section{Application to Ordinary Differential Equation}

In this section we present an example where Theorem 2.1 can be applied. This example is inspired in $[2,4,7]$.

Definition 3.1. Let $\mathfrak{B}$ denote the class of those functions $\phi:[0, \infty) \rightarrow[0, \infty)$ which satisfies the following condition:

(i) $\phi$ is increasing,

(ii) for each $x>0, \phi(x)<x$,

(iii) $\beta(x)=\phi(x) / x \in S$.

For example, $\phi(x)=a x$, where $0 \leq a<1, \phi(x)=x /(x+1)$, and $\phi(x)=\ln (1+x)$ are in $\mathfrak{B}$.

Theorem 3.2. Consider problem (1.1) with $f: I \times \mathbb{R} \rightarrow \mathbb{R}$ is continuous and suppose that there exists $\lambda>0$ such that for $x, y \in \mathbb{R}$ with $y \geq x$

$$
0 \leq f(t, y)+\lambda y-[f(t, x)+\lambda x] \leq \lambda \phi(y-x)
$$


where $\phi \in \mathfrak{B}$. Then the existence of a lower solution of (1.1) provides the existence of a unique solution of (1.1).

Proof. Problem (1.1) is equivalent to the integral equation

$$
u(t)=\int_{0}^{T} G(t, s)[f(s, u(s))+\lambda u(s)] d s,
$$

where

$$
G(t, s)= \begin{cases}\frac{e^{\lambda(T+s-t)}}{e^{\lambda T}-1}, & 0 \leq s<t \leq T \\ \frac{e^{\lambda(s-t)}}{e^{\lambda T}-1}, & 0 \leq t<s \leq T\end{cases}
$$

Define $F: C(I, \mathbb{R}) \rightarrow C(I, \mathbb{R})$ by

$$
(F u)(t)=\int_{0}^{T} G(t, s)[f(s, u(s))+\lambda u(s)] d s .
$$

Note that if $u \in C(I, \mathbb{R})$ is a fixed point of $F$ then $u \in C^{1}(I, \mathbb{R})$ is a solution of (1.1). Now, we check that hypotheses in Theorem 2.1 are satisfied. Indeed, $X=C(I, \mathbb{R})$ is a partially ordered set if we define the following order relation in $X$ :

$$
x, y \in C(I, \mathbb{R}), \quad x \leq y \quad \text { iff } x(t) \leq y(t), \quad \forall t \in I .
$$

The mapping $F$ is increasing since, by hypotheses, for $u \geq v$

$$
f(t, u)+\lambda u \geq f(t, v)+\lambda v
$$

which implies for $t \in I$, using that $G(t, s)>0$ for $(t, s) \in I \times I$, that

$$
\begin{aligned}
(F u)(t) & =\int_{0}^{T} G(t, s)[f(s, u(s))+\lambda u(s)] d s \\
& \geq \int_{0}^{T} G(t, s)[f(s, v(s))+\lambda v(s)] d s=(F v)(t) .
\end{aligned}
$$

Beside, for $u \geq v$

$$
\begin{aligned}
d(F u, F v) & =\sup _{t \in I}|(F u)(t)-(F v)(t)| \\
& \leq \sup _{t \in I} \int_{0}^{T} G(t, s)|f(s, u(s))+\lambda u(s)-(f(s, v(s))+\lambda v(s))| d s \\
& \leq \sup _{t \in I} \int_{0}^{T} G(t, s) \cdot \lambda \phi(u(s)-v(s)) d s
\end{aligned}
$$


As the function $\phi(x)$ is increasing and $u \geq v$ then $\phi(u(s)-v(s)) \leq \phi(d(u, v))$ we obtain

$$
\begin{aligned}
d(F u, F v) & \leq \sup _{t \in I} \int_{0}^{T} G(t, s) \cdot \lambda \phi(u(s)-v(s)) d s \\
& \leq \lambda \phi(d(u, v)) \sup _{t \in I} \int_{0}^{T} G(t, s) d s \\
& \left.\left.=\lambda \phi(d(u, v)) \sup _{t \in I} \frac{1}{e^{\lambda T}-1}\left(\frac{1}{\lambda} e^{\lambda(T+s-t)}\right]_{0}^{t}+\frac{1}{\lambda} e^{\lambda(s-t)}\right]_{t}^{T}\right) \\
& =\lambda \phi(d(u, v)) \cdot \frac{1}{\lambda\left(e^{\lambda T}-1\right)}\left(e^{\lambda T}-1\right)=\phi(d(u, v)) .
\end{aligned}
$$

Then for $u \geq v$

$$
d(F u, F v) \leq \alpha(d(u, v)) d(u, v) .
$$

Finally, let $\beta(t)$ be a lower solution of (1.1), and we will show that $\beta \leq F(\beta)$. Indeed,

$$
\beta^{\prime}(t)+\lambda \beta(t) \leq f(t, \beta(t))+\lambda \beta(t), \quad \text { for } t \in I .
$$

Multiplying by $e^{\lambda t}$ we get

$$
\left(\beta(t) e^{\lambda t}\right)^{\prime} \leq[f(t, \beta(t))+\lambda \beta(t)] e^{\lambda t}, \quad \text { for } t \in I,
$$

and this gives us

$$
\beta(t) e^{\lambda t} \leq \beta(0)+\int_{0}^{t}[f(s, \beta(s))+\lambda \beta(s)] e^{\lambda s} d s, \quad \text { for } t \in I
$$

which implies that

$$
\beta(0) e^{\lambda T} \leq \beta(T) e^{\lambda T} \leq \beta(0)+\int_{0}^{T}[f(s, \beta(s))+\lambda \beta(s)] e^{\lambda s} d s,
$$

and so

$$
\beta(0) \leq \int_{0}^{T} \frac{e^{\lambda s}}{e^{\lambda T}-1}[f(s, \beta(s))+\lambda \beta(s)] d s
$$

From this equality and (3.13) we obtain

$$
\beta(t) e^{\lambda t} \leq \int_{0}^{t} \frac{e^{\lambda(T+s)}}{e^{\lambda T}-1}[f(s, \beta(s))+\lambda \beta(s)] d s+\int_{t}^{T} \frac{e^{\lambda s}}{e^{\lambda T}-1}[f(s, \beta(s))+\lambda \beta(s)] d s,
$$


and, consequently,

$$
\beta(t) \leq \int_{0}^{t} \frac{e^{\lambda(T+s-t)}}{e^{\lambda T}-1}[f(s, \beta(s))+\lambda \beta(s)] d s+\int_{t}^{T} \frac{e^{\lambda(s-t)}}{e^{\lambda T}-1}[f(s, \beta(s))+\lambda \beta(s)] d s .
$$

Hence

$$
\beta(t) \leq \int_{0}^{T} G(t, s)[f(s, \beta(s))+\lambda \beta(s)] d s=(F(\beta))(t), \quad \text { for } t \in I
$$

Finally, Theorem 2.1 gives that $F$ has a unique fixed point.

Example 3.3. Let $\phi_{0}:[0, \infty) \rightarrow[0, \infty)$ be a defined

$$
\phi_{0}(t)= \begin{cases}0, & 0 \leq t \leq 3 \\ 3 t-9, & 3<t \leq 4 \\ \frac{3}{4} t, & 4<t\end{cases}
$$

Let $f: I \times \mathbb{R} \rightarrow \mathbb{R}$ be continuous and suppose that there exists $\lambda>0$ such that for $x, y \in \mathbb{R}$ with $y \geq x$

$$
0 \leq f(t, y)+\lambda y-[f(t, x)+\lambda x] \leq \lambda \phi_{0}(y-x)
$$

Then be Theorem 2.1, the existence of a lower solution for (1.1) provides the existence of a unique solution of (1.1).

The example discussed above cannot be the result of Harjani and Sadarangani noted Theorem 1.2 , because $\psi(x)=x-\phi_{0}(x)$ is not increasing.

\section{Acknowledgment}

The third author of this work was partially supported by Basic Science Research Program through the National Research Foundation of Korea (NRF) funded by the Ministry of Education, Science and Technology (Grant no.: 2010-0010243).

\section{References}

[1] R. P. Agarwal, M. A. El-Gebeily, and D. O'Regan, "Generalized contractions in partially ordered metric spaces," Applicable Analysis, vol. 87, no. 1, pp. 109-116, 2008.

[2] A. Amini-Harandi and H. Emami, "A fixed point theorem for contraction type maps in partially ordered metric spaces and application to ordinary differential equations," Nonlinear Analysis, vol. 72 no. 5, pp. 2238-2242, 2010.

[3] T. Gnana Bhaskar and V. Lakshmikantham, "Fixed point theorems in partially ordered metric spaces and applications," Nonlinear Analysis, vol. 65, no. 7, pp. 1379-1393, 2006.

[4] J. Harjani and K. Sadarangani, "Fixed point theorems for weakly contractive mappings in partially ordered sets," Nonlinear Analysis, vol. 71, no. 7-8, pp. 3403-3410, 2009. 
[5] V. Lakshmikantham and L. B. Cirić, "Coupled fixed point theorems for nonlinear contractions in partially ordered metric spaces," Nonlinear Analysis, vol. 70, no. 12, pp. 4341-4349, 2009.

[6] J. J. Nieto, R. L. Pouso, and R. Rodríguez-López, "Fixed point theorems in ordered abstract spaces," Proceedings of the American Mathematical Society, vol. 135, no. 8, pp. 2505-2517, 2007.

[7] J. J. Nieto and R. Rodríguez-López, "Contractive mapping theorems in partially ordered sets and applications to ordinary differential equations," Order, vol. 22, no. 3, pp. 223-239, 2005.

[8] A. C. M. Ran and M. C. B. Reurings, "A fixed point theorem in partially ordered sets and some applications to matrix equations," Proceedings of the American Mathematical Society, vol. 132, no. 5, pp. 1435-1443, 2004.

[9] J. J. Nieto and R. Rodríguez-López, "Existence and uniqueness of fixed point in partially ordered sets and applications to ordinary differential equations," Acta Mathematica Sinica, vol. 23, no. 12, pp. 2205$2212,2007$. 


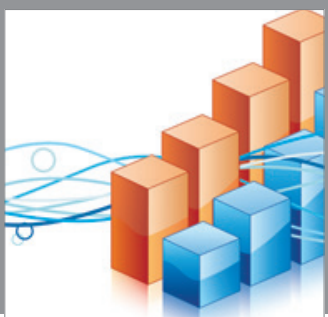

Advances in

Operations Research

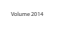

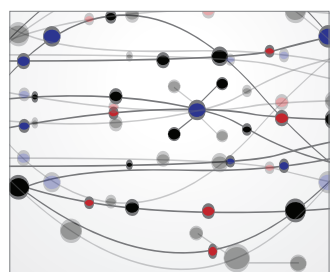

\section{The Scientific} World Journal
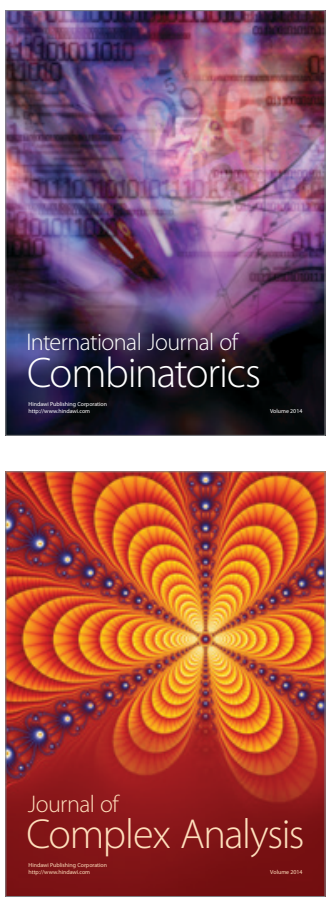

International Journal of

Mathematics and

Mathematical

Sciences
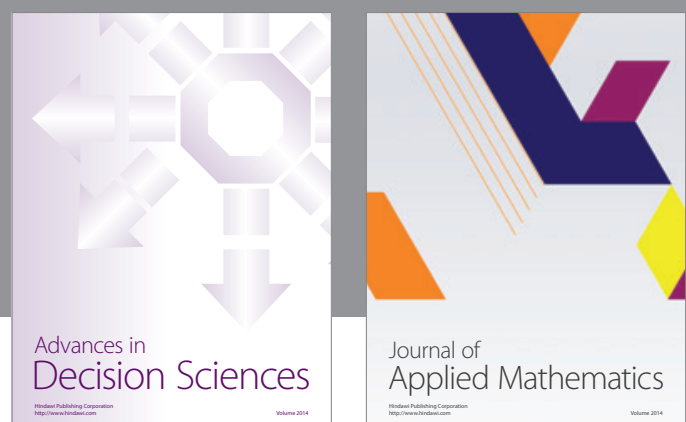

Journal of

Applied Mathematics
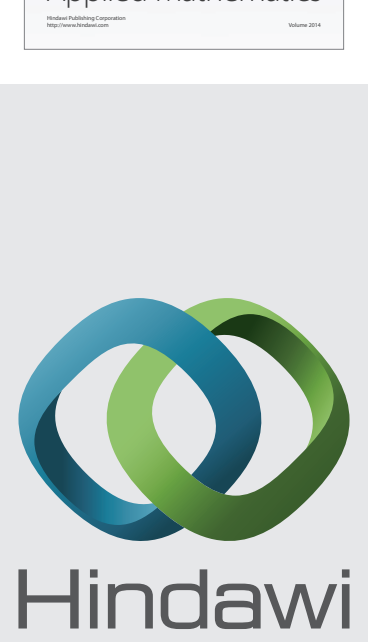

Submit your manuscripts at http://www.hindawi.com
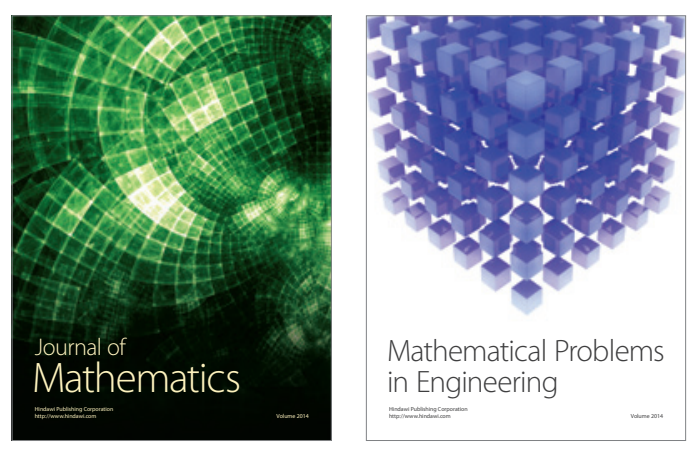

Mathematical Problems in Engineering
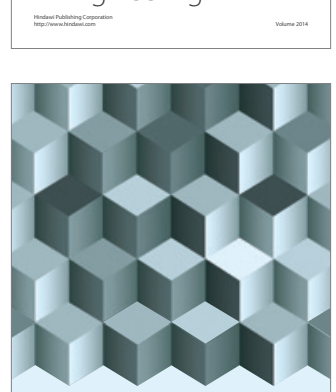

Journal of

Function Spaces
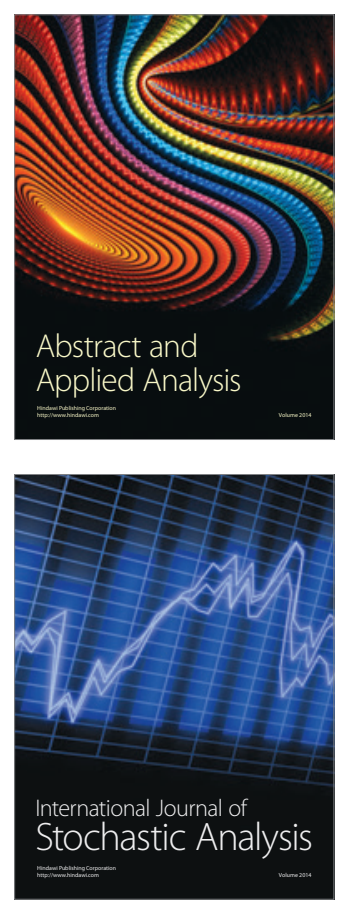

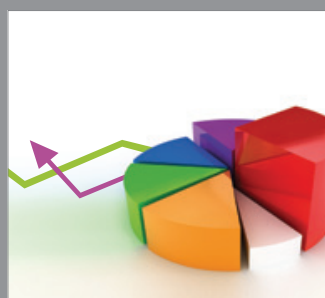

ournal of

Probability and Statistics

Promensencen
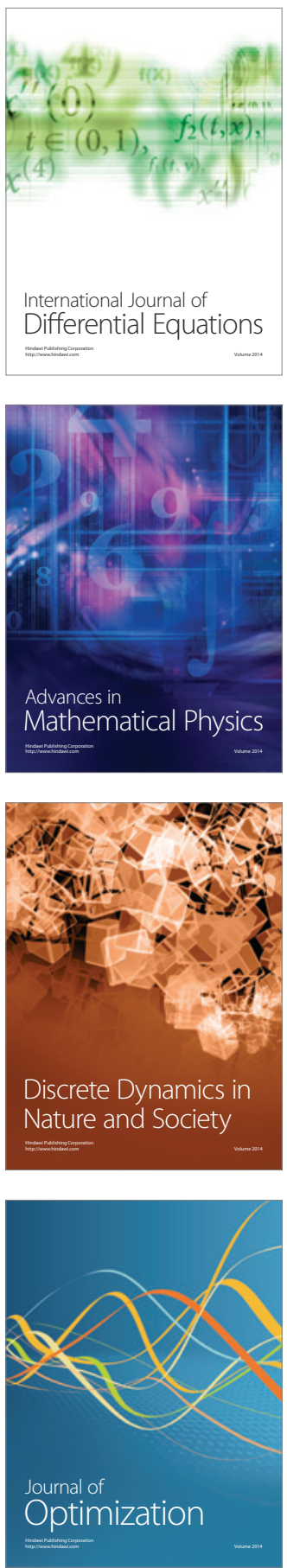